\title{
Increased NG2 and SOX2 expression is associated with high-grade choroid plexus tumors
}

\author{
PENG ZHAO $^{1}$, ZICHAO FENG ${ }^{1}$, QICHAO QI $^{1}$, BIN HUANG $^{1}$, \\ ANJING CHEN $^{1}$, XINGANG LI ${ }^{1}$, XINYU WANG ${ }^{1}$ and JIAN WANG ${ }^{1,2}$ \\ ${ }^{1}$ Department of Neurosurgery, Qilu Hospital of Shandong University and Brain Science Research Institute, Shandong \\ University, Jinan, Shandong 250012, P.R. China; ${ }^{2}$ Department of Biomedicine, University of Bergen, 5009 Bergen, Norway
}

Received June 8, 2016; Accepted March 31, 2017

DOI: $10.3892 / 01.2017 .6326$

\begin{abstract}
The World Health Organization classification of choroid plexus tumors (CPT) includes three distinct grades: Choroid plexus papilloma (CPP), atypical choroid plexus papilloma (ACPP) and choroid plexus carcinoma (CPC). The molecular basis for these pathological distinctions may help to stratify tumors and provide an insight into the clinical behavior of CPTs. In the present study, the progenitor and stem cell markers neuron glia antigen-2 (NG2) and sex-determining region Y-box 2 (SOX2) were investigated as potential biomarkers that may distinguish between distinct CPT grades. Immunohistochemistry was used to determine the expression of NG2 and SOX2 in CPTs $(n=34)$ from Chinese patients (21 males and 13 females) with a mean age of 31.1 years (range, 1-63 years). The proportion of cells stained were scored using a scale between 0 and $3+$, where 0 represents no staining and $3+$ represents strong staining, and mean scores for each marker were determined on the basis of tumor grade. Pathological diagnosis revealed a distribution of cases as follows: CPP, 25; ACPP, 5; and CPC, 4. NG2 and SOX2 were expressed in CPTs of all grades. The mean labeling indices for CPP, ACPP and CPC were 1.12, 1.80 and 2.75 for NG2, respectively, and 1.20, 2.00 and 3.00 for SOX2, respectively. Statistical analysis of the mean labeling indices revealed a significant association between the expression of NG2 and SOX2 and CPT grade $(\mathrm{P}=0.001$ and $<0.001$ for $\mathrm{CPP} / \mathrm{ACPP}$ and CPP/CPC, respectively). The results of the present study indicated that increased expression of NG2 and SOX2 was associated with higher-grade tumors and that these markers may be useful in determining CPT grade.
\end{abstract}

Correspondence to: Dr Xinyu Wang or Dr Jian Wang, Department of Neurosurgery, Qilu Hospital of Shandong University and Brain Science Research Institute, Shandong University, 107 Wenhua Xi Road, Jinan, Shandong 250012, P.R. China

E-mail: sjwwxy@126.com

E-mail: jian.wang@biomed.uib.no

Key words: choroid plexus tumors, immunohistochemical staining, neural/glial antigen 2, sex-determining region Y-box 2, tumor grade

\section{Introduction}

Choroid plexus tumors (CPTs) are rare intracranial neoplasms that derive from the choroid plexus epithelium of the ventricles $(1,2)$. The lesions are commonly located in the lateral and the fourth ventricles, and rarely in the cerebellopontine angle and the third ventricle $(3,4)$. The majority of CPTs arise with clinical symptoms that are associated with hydrocephalus as a result of direct mechanical obstruction of the flow of cerebrospinal fluid (CSF) and arachnoid granulation blockage from hemorrhage and overproduction of CSF $(1,5)$.

Treatment of CPTs is currently based on histological diagnosis. According to the World Health Organization (WHO) classification scheme, CPTs are diagnosed as choroid plexus papilloma (CPP, WHO grade I), atypical choroid plexus papilloma (ACPP, WHO grade II) and choroid plexus carcinoma (CPC, WHO grade III) (3). For all classifications, the general treatment strategy is surgical resection unless this is not possible due to tumor location, for example. Gross total resection (GTR) is the indicated course of treatment for CPP as well as for ACPP. CPP is a benign neoplasm and recurrence following GTR is rare, therefore adjuvant radiotherapy and chemotherapy are not generally recommended $(2,3,6,7)$. ACPP cases exhibit atypical histological features along with increased mitotic activity; however, patients with this type of tumor also exhibit a good prognosis following GTR. Patients with ACPP generally only require close observation and follow-up; however, chemotherapy is necessary when GTR is not performed or there is tumor dissemination or recurrence. CPC, however, is hypervascular and infiltrative, making GTR difficult to achieve. Patients with CPC may therefore require more comprehensive treatment, regardless of the extent of resection (8-12).

Radiotherapy and chemotherapy effectively prolong overall survival times in patients with CPC $(10,13,14)$. Radiotherapy, however, is only suitable for patients $>3$ years (2) and it remains unclear which patients may benefit from adjuvant therapy based on histological examination alone. Therefore, in the present study it was analyzed whether biomarkers may be used to predict and distinguish distinctions in prognosis between CPT cases of distinct grades. The association between the expression of progenitor and stem cell markers neuron glia antigen-2 (NG2) and sex-determining region Y-box 2 (SOX2) in CPTs and tumor grade was determined. 


\section{Materials and methods}

Ethical statement. The present study was approved by the Ethics Committee of Qilu Hospital, Shandong University (Jinan, China) and all procedures were conducted according to the World Medical Association Declaration of Helsinki Ethical Principles for Medical Research Involving Human Subjects (2013). Written informed consent was obtained from all study participants.

Patients. Patients $(n=34)$ treated for pathologically confirmed CPT in the Department of Neurosurgery, Qilu Hospital of Shandong University, between January 2003 and December 2013 were included in the present study. Tumors were resected using microscopic surgery, with the surgical approach based on tumor location and size in order to avoid damage to cortical functional areas and tracts. Patients received routine post-surgical care and follow-up. Surgical specimens were formalin-fixed, paraffin-embedded, sectioned and stained with hematoxylin and eosin for histological examination by a neuropathologist. Patient characteristics are provided in Table I.

Immunohistochemistry. Paraffin-embedded specimens were sectioned $(3 \mu \mathrm{m})$, deparaffinized in xylene and rehydrated in a graded series of alcohol. Antigen retrieval was carried out by heating sections in a microwave oven in citrate buffer ( $\mathrm{pH}$ 6.0; Thermo Fisher Scientific, Inc., Waltham, MA, USA; 005000) at $97^{\circ} \mathrm{C}$ for $20 \mathrm{~min}$ and then allowing them to cool to room temperature (RT). Endogenous peroxidase activity was inhibited by incubation at RT with $3 \%$ $\mathrm{H}_{2} \mathrm{O}_{2}$ for $20 \mathrm{~min}$ and subsequently incubated at $4^{\circ} \mathrm{C}$ with primary antibodies overnight (anti-SOX2; ab97959; 1:1,000; anti-NG2; ab50009; 1:50; Abcam, Cambridge, MA, USA). Positive and negative controls were included in parallel. Detection was performed by incubating slides at RT for 30 min with a biotinylated secondary antibody followed by streptavidin-biotin-horseradish peroxidase complex (SP9000 kit; Beijing Zhongshan Jinqiao Biotechnology Co., Ltd., Beijng, China; anti-mouse/rat/rabbit, Beijing, China). All washes in between incubations were performed with PBS and diaminobenzidine (DAB) was used as the chromogenic substrate for visualization. Sections were counterstained with hematoxylin and eosin, followed by differentiation in hydrochloric acid-ethanol and bluing in ammonia water. Slides were dehydrated in an ethanol series, cleared with xylene, mounted in neutral balsam and examined under a light microscope (Leica Application Suite version 4, Wetzlar, Germany). Slides were scored between 0 and $3+$ on the basis of the proportion of NG2- or SOX2-positive neoplastic cells ( 0 , no staining; $1+,<25 ; 2+, 26$ to 50 ; and $3+,>50 \%)$.

Statistical analysis. Statistical analysis was performed using SPSS software (version 19.0 for Windows; IBM Corp., Armonk, NY, USA). The association between age and tumor grade was analyzed using Student's t-test and Fisher's exact test. The association between sex, NG2/SOX2 labeling index and tumor grade were examined using $\chi^{2}$ or Fisher's exact tests. $\mathrm{P}<0.05$ was considered to indicate a statistically significant difference.
Table I. Clinicopathological characteristics of 34 patients with choroid plexus tumors of various grades.

\begin{tabular}{lcccc}
\hline Characteristic & All & CPP & ACPP & CPC \\
\hline $\begin{array}{l}\text { No. of patients } \\
\text { Age, years }\end{array}$ & 34 & 25 & 5 & 4 \\
$\quad$ Mean & 31.1 & 30.5 & 32.5 & 28.8 \\
$\quad$ Range & $1-63$ & $1-63$ & $2-61$ & $1-53$ \\
Sex & & & & \\
Male & 21 & 15 & 4 & 2 \\
Female & 13 & 10 & 1 & 2 \\
Location of tumor & & & & \\
$\quad$ Lateral ventricle & 14 & 7 & 4 & 3 \\
$\quad$ Third ventricle & 1 & 1 & 0 & 0 \\
Fourth ventricle & 15 & 13 & 1 & 1 \\
Cerebellopontine angle & 4 & 4 & 0 & 0 \\
\hline
\end{tabular}

CPP, choroid plexus papilloma; ACPP, atypical choroid plexus papilloma; CPC, choroid plexus carcinoma.

\section{Results}

Clinicopathological features. Clinical characteristics of patients are presented in Table I. A total of 34 patients ( 21 males and 13 females) were included in the present study and the mean age was 31.1 years (range, 1-63 years). All patients underwent surgical resection and all tumor grades were included in the cohort [CPP $(n=25)$, ACPP $(n=5)$ and CPC $(n=4)]$. Atypical morphology was observed only in high-grade CPTs (Fig. 1). Tumor location was determined on the basis of radiographic studies and surgical results, and was distributed as follows: Lateral ventricle $(n=14)$, third ventricle $(n=1)$, fourth ventricle $(n=5)$ and cerebellopontine angle $(n=4)$. Notably, no association was identified between tumor grade and age or any other clinicopathological factor examined.

Overexpression of NG2 and SOX2 is associated with higher grade in CPT. Immunohistochemistry was performed to determine whether CPT cases expressed NG2 or SOX2 and, if so, whether expression was associated with the tumor grade. The results of the present study demonstrated that NG2 and SOX2 were expressed in CPT. SOX2 and NG2 expression was identified in 34 and 33 cases, respectively (Figs. 2 and 3, respectively). Staining for NG2 and SOX2 were scored for each slide. The mean labeling indices for CPP, ACPP and CPC were 1.12, 1.80 and 2.75, respectively, for NG2, and 1.20, 2.00 and 3.00, respectively, for SOX2.

A score $\geq 2$ for immunohistochemistry was considered to indicate increased expression. The distribution of cases with scores $\geq 2$ for NG2 and SOX 2 was as follows: CPP, 3/25 and 4/25; ACPP, 4/5 and 4/5; and CPC, 4/4 and 4/4, respectively. Almost all the cases exhibiting decreased expression of NG2 or SOX 2 were CPP and a $\chi^{2}$ test was therefore used to identify the association between expression and tumor grade, in order to determine their utility as biomarkers in pathological diagnosis. Statistically significant associations were identified between 

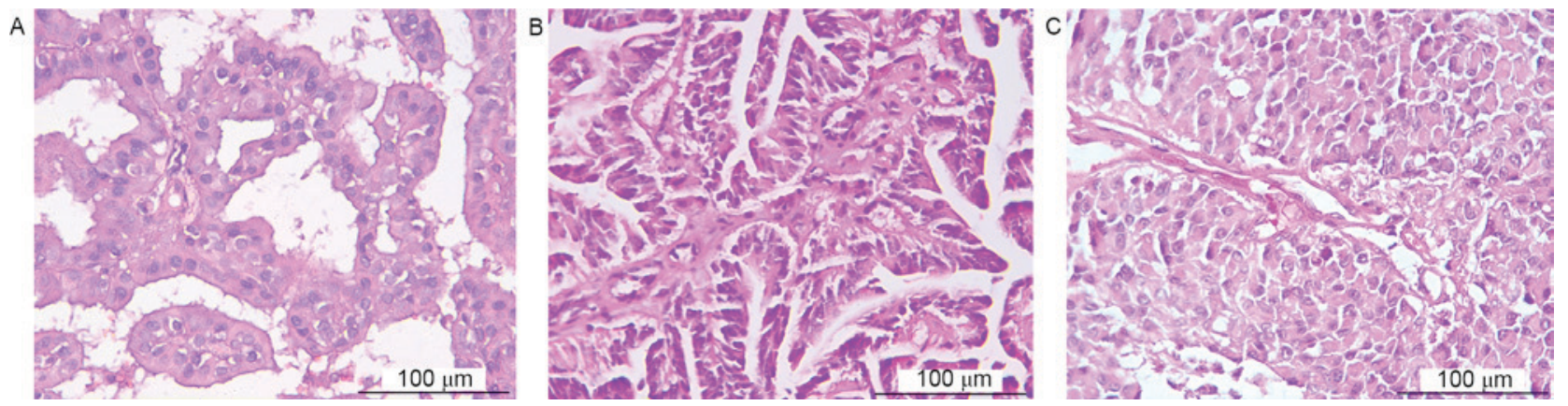

Figure 1. Pathological features of distinct World Health Organization graded choroid plexus tumors. Routine hematoxylin and eosin staining of tissue sections $(3 \mu \mathrm{m})$ from choroid plexus tumors representing distinct World Health Organization grades. (A) Choroid plexus papilloma, (B) atypical choroid plexus papilloma and (C) choroid plexus carcinoma (magnification, $\mathrm{x} 40$ ).
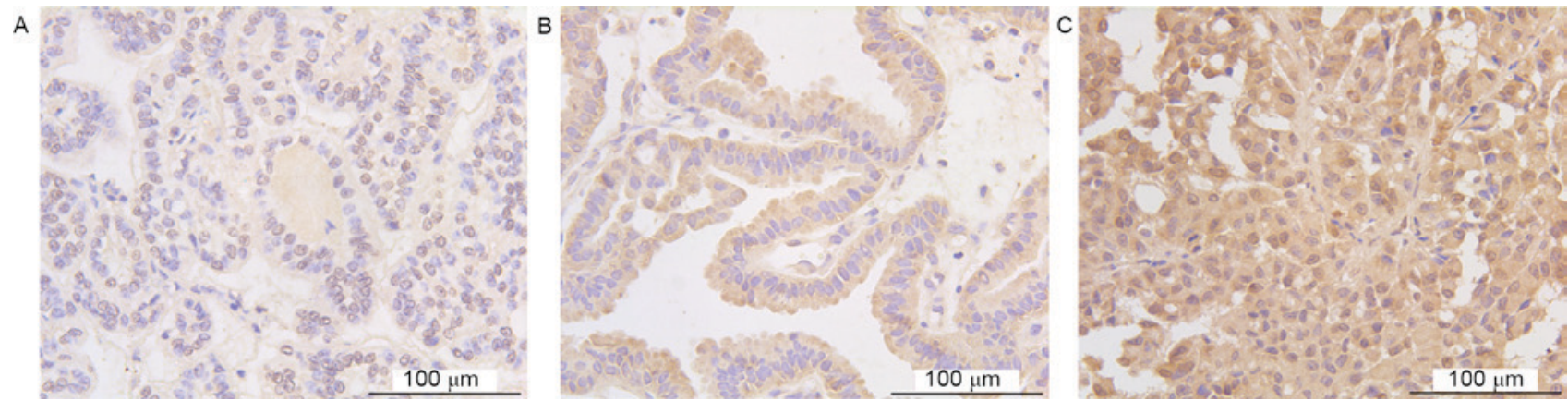

Figure 2. Increased expression of NG2 in higher-grade choroid plexus tumors. Immunohistochemical staining of NG2 in tissue sections representing (A) choroid plexus papilloma, (B) atypical choroid plexus papilloma and (C) choroid plexus carcinoma (magnification, $\mathrm{x} 40$ ).
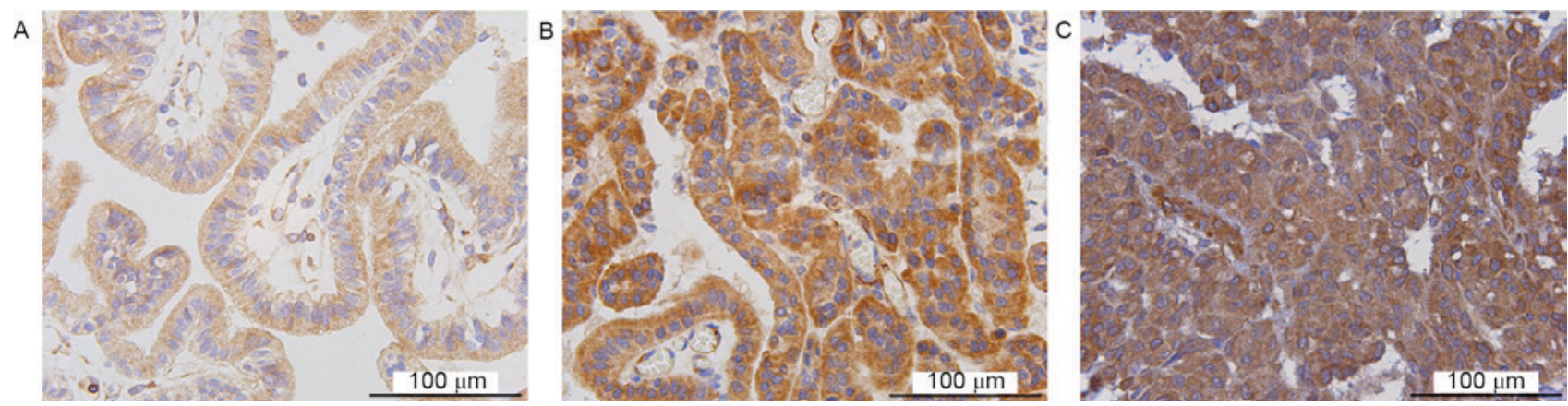

Figure 3. Increased expression of SOX2 in higher-grade choroid plexus tumors. Immunohistochemical staining of SOX2 in tissue sections representing (A) choroid plexus papilloma, (B) atypical choroid plexus papilloma and (C) choroid plexus carcinoma (magnification, x40).

CPP and ACPP for NG2 and SOX2 $(\mathrm{P}=0.001$ and 0.003 , respectively) in addition to $\mathrm{CPP}$ and $\mathrm{CPC}(\mathrm{P}<0.001)$. However, analysis for the comparison between ACPP and CPC cases were not statistically significant ( $\mathrm{P}=0.343$ for $\mathrm{NG} 2$ and $\mathrm{SOX} 2)$. These results demonstrated that expression of NG2 and SOX2 were significantly associated with tumor grade and that increased expression may be used to distinguish between grades of CPP and ACPP and between CPP and CPC (Table II).

\section{Discussion}

Molecular markers serve an integral role in current cancer diagnosis, providing a basis for distinguishing between the clinical behavior of tumors that may otherwise appear pathologically similar. In the present study, the expression of progenitor and stem cell markers NG2 and SOX2 in distinct grades of CPTs were examined using immunohistochemical staining. The results of the present study revealed a statistically significant association between increased expression of NG2 and SOX2 and high-grade CPT. These results indicate that NG2 and SOX2 may refine the diagnosis and development of treatment strategies of affected patients.

To the best of our knowledge there are no previous studies investigating NG2 or SOX2 expression levels in CPTs but they have been previously associated with cancer development (15-22). Increased expression of NG2 has been demonstrated in a variety of head and neck cancers, glioma and pituitary cells (15-18). SOX2 overexpression or gene amplification has been associated with the development of lung, esophageal, breast and gastric cancers (19-22). 
Table II. Comparison of immunohistochemical score between various grades of patients with choroid plexus tumors.

\begin{tabular}{lcccr}
\hline & \multicolumn{3}{c}{ WHO grade } & \\
\cline { 2 - 4 } Variable & I & II & III & P-value \\
\hline NG2 & & & & $0.001^{\mathrm{a}}$ \\
0 & 1 & 0 & 0 & $0.343^{\mathrm{b}}$ \\
1 & 21 & 1 & 0 & $<0.001^{\mathrm{c}}$ \\
2 & 2 & 4 & 1 & \\
3 & 1 & 0 & 3 & \\
Expression score $\geq 2$ & 3 & 4 & 4 & \\
Mean score & 1.12 & 1.80 & 2.75 & \\
SOX2 & & & & \\
0 & 0 & 0 & 0 & \\
1 & 21 & 1 & 0 & $0.003^{\mathrm{a}}$ \\
2 & 3 & 3 & 0 & $0.343^{\mathrm{b}}$ \\
3 & 1 & 1 & 4 & $<0.001^{\mathrm{c}}$ \\
Expression score $\geq 2$ & 4 & 4 & 4 & \\
Mean score & 1.20 & 2.00 & 3.00 & \\
\hline
\end{tabular}

aI vs. II; ' $\mathrm{bI}$ vs. III; ' ${ }^{\mathrm{I}}$ vs. III. WHO, World Health Organization; NG2, neuron glia antigen-2; SOX2, sex-determining region Y-box 2.

NG2 expression is typically increased in the developing and adult central nervous system (CNS). It is a type-I membrane protein expressed by diverse cell types within the CNS during development and differentiation. NG2 has been identified to be expressed during development in extraneural progenitor cell types, including mesenchymal stem cells, chondroblasts, osteoblasts, immature keratinocytes, muscle progenitors and melanocytes (23). Additionally, NG2 serves a role in cell viability and angiogenesis (24). NG2 deficiency during early development results in the loss of pericyte-endothelium association and defective formation of basement membranes in blood vessels (25). NG2 has been postulated to function as a cell adhesion molecule, which was supported by the observation that the extracellular region between the transmembrane domain and the laminin G/neurexin/sex-hormone-binding globulin domains binds to integrins and collagen $\mathrm{V}$ and VI (26). Previous studies have revealed a more defined role for NG2 in brain homeostasis including intracellular functions in oligodendrocyte progenitor cells, which modulate migration, gene expression and $\alpha$-amino-3-hydroxy-5-methyl-4-isoxazol epropionic acid receptor clustering $(15,24,27)$.

SOX2 is classified in the SoxB1 family due to the similarity of the amino acid sequence of the high-mobility group box domain $(28,29)$. SOX 2 is a transcription factor that serves a primary role in maintaining stem cells in adult tissues, including the CNS. During development of the peripheral nervous system, SOX 2 is expressed in neural crest stem cells and regulates differentiation into dorsal root ganglion neurons. In addition, SOX2 is expressed in immature Schwann cells and inhibits myelination (30) and is re-expressed in undifferentiated myelinating Schwann cells following nerve injury and regulates their sorting. Finally, it is one of the transcription factors initially used to generate induced pluripotent stem cells from fibroblast cells (31). Thus, NG2 and SOX2 function in the maintenance of progenitor and stem cells, which may contribute to the development of cancer.

NG2 and SOX2 exhibit increased expression in higher-grade CPTs. Although additional studies are required to elucidate the functional roles of NG2 and SOX2 in the development of CPTs, the results of the present study demonstrate that expression levels of these proteins may be helpful for determining tumor grade in histologically controversial cases. In addition, NG2 and SOX2 may assist in identifying cases where aggressive or novel treatment may be necessary for improved patient outcomes.

\section{Acknowledgements}

The present study was supported by the Natural Science Foundation of China (grant nos. 81402060 and 81572487), the Shandong Provincial Natural Science Foundation (grant nos. BS2014YY033 and BS2012YY016), the Special foundation for Taishan Scholars (grant nos. ts20110814 and tshw201502056), the Fundamental Research Funds of Shandong University, the Department of Science and Technology of Shandong Province (grant nos. 2015GGE27101 and 2015ZDXX0801A01), the China Postdoctoral Science Foundation (grant no. 2014M551916), the University of Bergen, Helse Bergen, Norway, and the Norwegian Centre for International Cooperation in Education (SIU) (grant no. UTF-2014/10047).

\section{References}

1. Strojan P, Popović M, Surlan K and Jereb B: Choroid plexus tumors: A review of 28-year experience. Neoplasma 51: 306-312, 2004.

2. Sun MZ, Oh MC, Ivan ME, Kaur G, Safaee M, Kim JM, Phillips JJ, Auguste KI and Parsa AT: Current management of choroid plexus carcinomas. Neurosurg Rev 37: 179-192; discussion 192, 2014.

3. Koh EJ, Wang KC, Phi JH, Lee JY, Choi JW, Park SH, Park KD, Kim IH, Cho BK and Kim SK: Clinical outcome of pediatric choroid plexus tumors: Retrospective analysis from a single institute. Childs Nerv Syst 30: 217-225, 2014.

4. Krishnan S, Brown PD, Scheithauer BW, Ebersold MJ, Hammack JE and Buckner JC: Choroid plexus papillomas: A single institutional experience. J Neurooncol 68: 49-55, 2004.

5. Buxton $\mathrm{N}$ and Punt J: Choroid plexus papilloma producing symptoms by secretion of cerebrospinal fluid. Pediatr Neurosurg 27: 108-111, 1997.

6. Samuel TA, Parikh J, Sharma S, Giller CA, Sterling K, Kapoor S, Pirkle $\mathrm{C}$ and Jillella A: Recurrent adult choroid plexus carcinoma treated with high-dose chemotherapy and syngeneic stem cell (bone marrow) transplant. J Neurol Surg A Cent Eur Neurosurg 74 (Suppl 1): e149-e154, 2013.

7. Wolff JE, Sajedi M, Brant R, Coppes MJ and Egeler RM: Choroid plexus tumours. Br J Cancer 87: 1086-1091, 2002.

8. Bettegowda C, Adogwa O, Mehta V, Chaichana KL, Weingart J, Carson BS, Jallo GI and Ahn ES: Treatment of choroid plexus tumors: A 20-year single institutional experience. J Neurosurg Pediatr 10: 398-405, 2012.

9. Kubicky CD, Sahgal A, Chang EL and Lo SS: Rare primary central nervous system tumors. Rare Tumors 6: 5449, 2014.

10. McEvoy AW, Harding BN, Phipps KP, Ellison DW, Elsmore AJ, Thompson D, Harkness W and Hayward RD: Management of choroid plexus tumours in children: 20 Years experience at a single neurosurgical centre. Pediatr Neurosurg 32: 192-199, 2000.

11. Rickert CH and Paulus W: Tumors of the choroid plexus. Microsc Res Tech 52: 104-111, 2001.

12. Turkoglu E, Kertmen H, Sanli AM, Onder E, Gunaydin A, Gurses L, Ergun BR and Sekerci Z: Clinical outcome of adult choroid plexus tumors: Retrospective analysis of a single institute. Acta Neurochir (Wien) 156: 1461-1478, 2014. 
13. Kourbeti IS, Jacobs AV, Koslow M, Karabetsos D and Holzman RS: Risk factors associated with postcraniotomy meningitis. Neurosurgery 60: 317-325; discussion 325-316, 2007.

14. O'Malley MR and Haynes DS: Assessment and management of meningitis following cerebellopontine angle surgery. Curr Opin Otolaryngol Head Neck Surg 16: 427-433, 2008.

15. Farnedi A, Rossi S, Bertani N, Gulli M, Silini EM, Mucignat MT, Poli T, Sesenna E, Lanfranco D, Montebugnoli L, et al: Proteoglycan-based diversification of disease outcome in head and neck cancer patients identifies NG2/CSPG4 and syndecan-2 as unique relapse and overall survival predicting factors. BMC Cancer 15: 352, 2015.

16. Nicolosi PA, Dallatomasina A and Perris R: Theranostic impact of NG2/CSPG4 proteoglycan in cancer. Theranostics 5: 530-544, 2015.

17. Tateno T, Nakano-Tateno T, Ezzat S and Asa SL: NG2 targets tumorigenic $\mathrm{Rb}$ inactivation in Pit1-lineage pituitary cells. Endocr Relat Cancer 23: 445-456, 2016.

18. Yadavilli S, Hwang EI, Packer RJ and Nazarian J: The role of NG2 proteoglycan in glioma. Transl Oncol 9: 57-63, 2016.

19. Carrasco-Garcia E, Santos JC, Garcia I, Brianti M, García-Puga M, Pedrazzoli J Jr, Matheu A and Ribeiro ML: Paradoxical role of SOX2 in gastric cancer. Am J Cancer Res 6 : 701-713, 2016.

20. Li Q, Liu F, Zhang Y, Fu L, Wang C, Chen X, Guan S and Meng X: Association of SOX2 and nestin DNA amplification and protein expression with clinical features and overall survival in non-small cell lung cancer: A systematic review and meta-analysis. Oncotarget 7: 34520-34531, 2016.

21. Schaefer T, Wang H, Mir P, Konantz M, Pereboom TC, Paczulla AM, Merz B, Fehm T, Perner S, Rothfuss OC, et al: Molecular and functional interactions between AKT and SOX2 in breast carcinoma. Oncotarget 6: 43540-43556, 2015.

22. van Olphen S, Biermann K, Spaander MC, Kastelein F, Steyerberg EW, Stoop HA, Bruno MJ and Looijenga LH: SOX2 as a novel marker to predict neoplastic progression in Barrett's esophagus. Am J Gastroenterol 110: 1420-1428, 2015.
23. Stallcup WB: The NG2 proteoglycan: Past insights and future prospects. J Neurocytol 31: 423-435, 2002.

24. Sakry D and Trotter J: The role of the NG2 proteoglycan in OPC and CNS network function. Brain Res 1638: 161-166, 2016.

25. Huang FJ, You WK, Bonaldo P, Seyfried TN, Pasquale EB and Stallcup WB: Pericyte deficiencies lead to aberrant tumor vascularizaton in the brain of the NG2 null mouse. Dev Biol 344: 1035-1046, 2010

26. Fukushi J, Makagiansar IT and Stallcup WB: NG2 proteoglycan promotes endothelial cell motility and angiogenesis via engagement of galectin-3 and alpha3beta1 integrin. Mol Biol Cell 15: 3580-3590, 2004

27. Brekke C, Lundervold A, Enger PØ, Brekken C, Stålsett E, Pedersen TB, Haraldseth O, Krüger PG, Bjerkvig R and Chekenya M: NG2 expression regulates vascular morphology and function in human brain tumours. Neuroimage 29: 965-976, 2006.

28. Albright JE, Stojkovska I, Rahman AA, Brown CJ and Morrison BE: Nestin-positive/SOX2-negative cells mediate adult neurogenesis of nigral dopaminergic neurons in mice. Neurosci Lett 615: 50-54, 2016.

29. Rizzino A and Wuebben EL: Sox2/Oct4: A delicately balanced partnership in pluripotent stem cells and embryogenesis. Biochim Biophys Acta 1859: 780-791, 2016.

30. Le N, Nagarajan R, Wang JY, Araki T, Schmidt RE and Milbrandt J: Analysis of congenital hypomyelinating Egr2Lo/Lo nerves identifies Sox 2 as an inhibitor of Schwann cell differentiation and myelination. Proc Natl Acad Sci USA 102: 2596-2601, 2005.

31. Takahashi K, Tanabe K, Ohnuki M, Narita M, Ichisaka T, Tomoda $\mathrm{K}$ and Yamanaka S: Induction of pluripotent stem cells from adult human fibroblasts by defined factors. Cell 131: 861-872, 2007. 